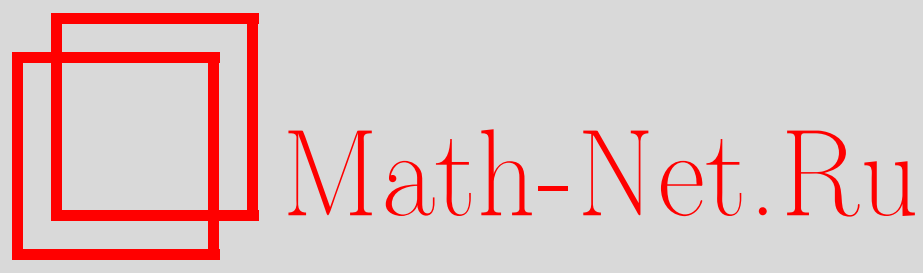

А. Мирза, М. Хассан, О свойствах интегрируемости суперсимметричной бездисперсионной интегрируемой системы со взаимодействием, ТМФ, 2018, том 195, номер 3, 381-390

DOI: https://doi.org/10.4213/tmf9498

Использование Общероссийского математического портала Math-Net.Ru подразумевает, что вы прочитали и согласны с пользовательским соглашением http://www.mathnet.ru/rus/agreement

Параметры загрузки:

IP : 54.198 .187 .58

26 апреля 2023 г., 03:48:34

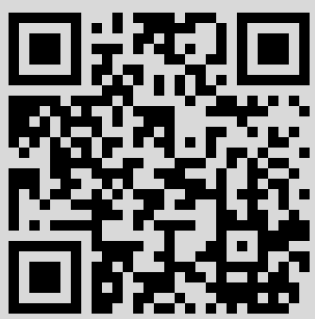




\title{
О СВОЙСТВАХ ИНТЕГРИРУЕМОСТИ СУПЕРСИММЕТРИЧНОЙ БЕЗДИСПЕРСИОННОЙ ИНТЕГРИРУЕМОЙ СИСТЕМЫ СО ВЗАИМОДЕЙСТВИЕМ
}

\begin{abstract}
Детально изучается проблема интегрируемости $N=1$ суперсимметричной бездисперсионной интегрируемой системы со взаимодействием. Представление Лакса для данной системы записано в виде $(3 \times 3)$-матрицы пары Лакса для суперполей и показано, что условие нулевой кривизны соответствует суперсимметричной бездисперсионной системе со взаимодействием. Из фермионного суперполевого представления Лакса выведены связанные уравнения Риккати для суперполей, с помощью которых затем получается бесконечный набор суперполевых сохраняющихся токов. Исследовано преобразование Дарбу рассматриваемой системы и путем его многократного применения получены многосолитонные решения.
\end{abstract}

Ключевые слова: интегрируемые системы, суперсимметрия, преобразование Дарбу, суперсимметричная бездисперсионная система со взаимодействием.

DOI: https://doi.org/10.4213/tmf9498

\section{1. ВВЕДЕНИЕ}

Суперсимметричные интегрируемые системы являются предметом многочисленных исследований, поскольку поиск новых решений суперсимметричных версий классических интегрируемых систем представляет большой интерес [1]-[6]. Для изучения различных свойств интегрируемых систем, которые также можно применить к суперсимметричным интегрируемым системам, были предложены многочисленные подходы, в частности билинейный метод Хироты, методы преобразования Беклунда и преобразования Дарбу, метод обратной задачи рассеяния и т. д. (см., например, монографию [7]). Преобразование Дарбу является эффективным подходом к нахождению солитонных решений различных интегрируемых систем, и в своем стандартном виде оно использовалось для многих суперсимметричных и классических интегрируемых уравнений [8]-[16].

*Department of Physics, University of the Punjab, Lahore, Punjab, Pakistan.

E-mail: arifa.mughal@gmail.com, mhassan.physics@pu.edu.pk 
В недавней статье [17] мы предложили $N=1$ суперсимметричное обобщение интегрируемой бездисперсионной системы со взаимодействием (далее мы называем ee SUSY-CD-системой, т. е. supersymmetric coupled dispersionless system). Мы получили уравнения для компонент поля, сводящиеся в бозонном пределе к обычной бездисперсионной системе. Мы также использовали билинейный метод Хироты для суперполя, чтобы получить мультисолитонные решения системы. Бездисперсионные системы со взаимодействием [18]-[27] составляют важный класс нелинейных интегрируемых систем, которые обладают многими интересными свойствами и имеют разнообразные применения в математике и физике (см., например, статью [17] и ссылки в ней).

Прежде чем двигаться дальше, напомним обозначения работы [17]. Мы определяем $N=1$ SUSY-CD-систему в терминах фермионных (грассманово-нечетных) суперполей $Q(x, t, \theta, \zeta)$ и $R(x, t, \theta, \zeta)$ :

$$
D_{x} D_{t} Q+R D_{x} R=0, \quad D_{x} D_{t} R-R D_{x} Q=0,
$$

где $\theta$ и $\zeta$ - антикоммутирующие грассмановы переменные $\left(\theta^{2}=0, \zeta^{2}=0\right)$. Ковариантные производные в суперпространстве определяются как $D_{x}=\partial_{\theta}+\theta \partial_{x}$, $D_{t}=\partial_{\zeta}+\zeta \partial_{t}$, при этом $D_{x}^{2}=\partial_{x}, D_{t}^{2}=\partial_{t}$ и оператор $D_{x}$ антикоммутирует с оператором $D_{t}$. Преобразования суперсимметрии порождаются операторами $Q_{1}=\partial_{\theta}-\theta \partial_{x}$ и $Q_{2}=\partial_{\zeta}-\zeta \partial_{t}$. Операторы $Q_{1}$ и $Q_{2}$ антикоммутируют с операторами ковариантного дифференцирования $D_{x}$ и $D_{t}$ соответственно.

Цель настоящей работы - изучить различные свойства интегрируемости интегрируемой SUSY-CD-системы. Следуя работе [28], мы записываем $(3 \times 3)$-матрицу представления Лакса для суперполей SUSY-CD-системы. Это представление Лакса приводит к двум парам суперполевых уравнений Риккати, которые затем используются для расчета бесконечного числа локальных сохраняющихся токов. В нашем анализе мы получаем преобразование Дарбу суперполей SUSY-CD-системы и строим мультисолитонные решения.

Статья организована следующим образом: В разделе 2 мы записываем представление Лакса для суперполей в виде матрицы размера $3 \times 3$. Известно, что условие нулевой кривизны для суперполевых операторов Лакса приводит к SUSY-CD-системе. В разделе 3 приводятся две пары уравнений Риккати для суперполей, которые получаются из линейной спектральной задачи. Эти уравнения используются для построения бесконечного числа суперполевых сохраняющихся токов. В разделе 4 мы изучаем преобразования Дарбу суперполей линейной спектральной задачи и суперполей SUSY-CD-системы и с его помощью рассчитываем $N$-солитонное решение SUSY-CD-системы. Раздел 5 содержит заключительные замечания.

\section{2. СУПЕРПОЛЕВОЕ ПРЕДСТАВЛЕНИЕ ЛАКСА ДЛЯ SUSY-CD-СИСТЕМЫ}

Чтобы линеаризовать SUSY-CD-систему, рассмотрим следующую линейную систему относительно суперполевой волновой функции $\Psi$ :

$$
\begin{aligned}
& D_{x} \Psi=\left(\mathcal { J } \left\{D_{x} Q(x, t, \theta, \zeta)\right.\right.\left.-i D_{x} R(x, t, \theta, \zeta)\right\}+ \\
&\left.+\mathcal{K}\left\{D_{x} Q(x, t, \theta, \zeta)+i D_{x} R(x, t, \theta, \zeta)\right\}\right) \Psi, \\
& D_{t} \Psi=(\mathcal{M} R(x, t, \theta, \zeta)+\mathcal{N}) \Psi
\end{aligned}
$$


где $\mathcal{J}, \mathcal{K}, \mathcal{M}$ и $\mathcal{N}$ суть комплекснозначные матрицы. Условие совместности уравнений (2) эквивалентно SUSY-CD-системе (1). Алгебраические связи между матрицами $\mathcal{J}, \mathcal{K}, \mathcal{M}$ и $\mathcal{N}$ задаются как

$$
i \mathcal{J}=[\mathcal{M}, \mathcal{J}], \quad i \mathcal{K}=[\mathcal{K}, \mathcal{M}], \quad \frac{i}{2} \mathcal{M}=\{\mathcal{K}, \mathcal{N}\}, \quad-\frac{i}{2} \mathcal{M}=\{\mathcal{J}, \mathcal{N}\}
$$

где $[\cdot, \cdot]$ - коммутатор и $\{\cdot, \cdot\}$ - антикоммутатор. Решение этих уравнений в алгебре Ли $\mathfrak{s l}(3, \mathfrak{C})$ имеет вид

$$
\begin{aligned}
\mathcal{J}=\frac{1}{2}\left(\begin{array}{rrr}
0 & 0 & -1 \\
0 & 0 & 0 \\
0 & -1 & 0
\end{array}\right), & \mathcal{K}=\frac{1}{2}\left(\begin{array}{lll}
0 & 0 & 0 \\
0 & 0 & 1 \\
1 & 0 & 0
\end{array}\right), \\
\mathcal{M}=\left(\begin{array}{rrr}
i & 0 & 0 \\
0 & -i & 0 \\
0 & 0 & 0
\end{array}\right), & \mathcal{N}=\left(\begin{array}{rrr}
0 & 0 & -1 \\
0 & 0 & 1 \\
-1 & 1 & 0
\end{array}\right) .
\end{aligned}
$$

Непараметрическую линейную задачу (2) можно привести к спектральной задаче, зависящей от спектрального параметра $\lambda$. Для этого применяется точечная симметрия Ли, как это сделано в работе [29]. Введем преобразование

$$
\tilde{x}=\lambda x, \quad \tilde{t}=\lambda^{-1} t, \quad \tilde{\theta}=\lambda^{1 / 2} \theta, \quad \tilde{\zeta}=\lambda^{-1 / 2} \zeta, \quad \lambda= \pm e^{\mu},
$$

где $\mu$ - произвольная бозонная постоянная в алгебре Грассмана. Уравнения (2) неинвариантны относительно преобразования (5), следовательно, линейная спектральная задача для SUSY-CD-системы (1) принимает вид

$$
D_{x} \Psi=A(x, t, \theta, \zeta ; \lambda) \Psi, \quad D_{t} \Psi=B(x, t, \theta, \zeta ; \lambda) \Psi,
$$

где $A(x, t, \theta, \zeta ; \lambda)$ и $B(x, t, \theta, \zeta ; \lambda)$ - суперполевые $(3 \times 3)$-матрицы, принимающие значения в супералгебре $\mathfrak{s l}(2 \mid 1, \mathcal{G})$, которые задаются как

$$
\begin{gathered}
A=\frac{1}{2 \sqrt{\lambda}}\left(\begin{array}{ccc}
0 & 0 & -\left(D_{x} Q-i D_{x} R\right) \\
0 & 0 & \left(D_{x} Q+i D_{x} R\right) \\
\left(D_{x} Q+i D_{x} R\right) & -\left(D_{x} Q-i D_{x} R\right) & 0
\end{array}\right), \\
B=\left(\begin{array}{ccc}
i R & 0 & -\sqrt{\lambda} \\
0 & -i R & \sqrt{\lambda} \\
-\sqrt{\lambda} & \sqrt{\lambda} & 0
\end{array}\right) .
\end{gathered}
$$

Условие совместности эквивалентно условию нулевой кривизны в суперпространстве

$$
D_{t} A+D_{x} B-\{A, B\}=0,
$$

которое, в свою очередь, эквивалентно SUSY-CD-системе (1). Чтобы сохранить градуировку, запишем суперполе $\Psi(x, t, \theta, \zeta)$ как трехкомпонентный вектор-столбец, содержащий грассманово-нечетные и четные функции. При этом $(2 \mid 1)$-супервектор $\Psi$ можно выразить как $\Psi=\left(\begin{array}{c}\psi \\ \phi \\ \chi\end{array}\right)$, где $\psi$ и $\phi-$ бозонные суперполя, а $\chi-$ фермионное суперполе. 
Мы также можем описать линейную спектральную задачу в ее бозонной версии. Используем свойства ковариантных производных $D_{x}$ и $D_{t}$ и запишем представление Лакса для SUSY-CD-системы:

$$
\begin{aligned}
& \partial_{x} \Psi=i\left(D_{x} A(x, t, \theta, \zeta ; \lambda)-(E A)^{2}\right) \Psi=U \Psi, \\
& \partial_{t} \Psi=i\left(D_{t} B(x, t, \theta, \zeta ; \lambda)-(E B)^{2}\right) \Psi=V \Psi,
\end{aligned}
$$

где $E=\operatorname{diag}(1,1,-1)$ и суперматрицы $U, V$ имеют вид

$$
\begin{gathered}
U=\left(\begin{array}{ccc}
-\frac{i}{4 \lambda}\left\{\left(D_{x} Q\right)^{2}-\left(i D_{x} R\right)^{2}\right\} & \frac{i}{4 \lambda}\left(D_{x} Q-i D_{x} R\right)^{2} & -\frac{i}{2 \sqrt{\lambda}}\left(\partial_{x} Q-i \partial_{x} R\right) \\
\frac{i}{4 \lambda}\left(D_{x} Q+i D_{x} R\right)^{2} & -\frac{i}{4 \lambda}\left\{\left(D_{x} Q\right)^{2}-\left(i D_{x} R\right)^{2}\right\} & \frac{i}{2 \sqrt{\lambda}}\left(\partial_{x} Q+i \partial_{x} R\right) \\
\frac{i}{2 \sqrt{\lambda}}\left(\partial_{x} Q+i \partial_{x} R\right) & -\frac{i}{2 \sqrt{\lambda}}\left(\partial_{x} Q-i \partial_{x} R\right) & \frac{i}{2 \lambda}\left\{\left(D_{x} Q\right)^{2}-\left(i D_{x} R\right)^{2}\right\}
\end{array}\right), \\
V=i\left(\begin{array}{ccc}
i D_{t} R+\lambda & -\lambda & i \sqrt{\lambda} R \\
-\lambda & -i D_{t} R+\lambda & i \sqrt{\lambda} R \\
-i \sqrt{\lambda} R & -i \sqrt{\lambda} R & 2 \lambda
\end{array}\right) .
\end{gathered}
$$

Условие совместности уравнений (9) эквивалентно классическому варианту условия нулевой кривизны в суперпространстве

$$
\partial_{t} U-\partial_{x} V+[U, V]=0
$$

которое дает SUSY-CD-систему (1).

Теперь введем калибровочное преобразование волновой функции и операторов Лакса, чтобы получить эквивалентное выражение для суперполевой пары Лакса. Это преобразование может оказаться полезным при выводе законов сохранения SUSY-CD-системы. Калибровочное преобразование собственной функции $\Psi$ определим как $\widetilde{\Psi}=G \Psi$, где калибровочная матрица имеет вид

$$
G=\left(\begin{array}{rrr}
-i & i & 0 \\
1 & 1 & 0 \\
0 & 0 & -i
\end{array}\right)
$$

Соответствующее преобразование операторов Лакса задается как

$$
\widetilde{A}=G A G^{-1}, \quad \widetilde{B}=G B G^{-1} .
$$

Таким образом, линейная система (7) становится калибровочно-эквивалентной следующей линейной системе:

$$
D_{x} \widetilde{\Psi}=\widetilde{A} \widetilde{\Psi}, \quad D_{t} \widetilde{\Psi}=\widetilde{B} \widetilde{\Psi},
$$

где

$$
\widetilde{A}=\left(\begin{array}{ccc}
0 & 0 & -\frac{1}{\sqrt{\lambda}} D_{x} Q \\
0 & 0 & -\frac{1}{\sqrt{\lambda}} D_{x} R \\
\frac{1}{2 \sqrt{\lambda}} D_{x} Q & \frac{1}{2 \sqrt{\lambda}} D_{x} R & 0
\end{array}\right), \quad \widetilde{B}=\left(\begin{array}{ccc}
0 & R & -2 \sqrt{\lambda} \\
-R & 0 & 0 \\
-\sqrt{\lambda} & 0 & 0
\end{array}\right)
$$

Условие $D_{t} \widetilde{A}+D_{x} \widetilde{B}-\{\widetilde{A}, \widetilde{B}\}=0$ эквивалентно уравнениям (1) для суперполей. 


\section{3. ЗАКОНЫ СОХРАНЕНИЯ SUSY-CD-СИСТЕМЫ}

Следуя работам [30]-[32], получим бесконечный набор суперполевых сохраняющихся токов для SUSY-CD-системы. Законы сохранения выводятся путем задания анзаца, в результате которого суперполевое уравнение непрерывности вытекает из уравнений Риккати для суперполей. Проведем следующие рассуждения.

Начнем с суперполевой собственной функции для пары Лакса (12): $\Psi=\left(\begin{array}{l}\Psi_{1} \\ \Psi_{2} \\ \Psi_{3}\end{array}\right)$, где $\Psi_{1}, \Psi_{2}$ - бозонные суперполя, а $\Psi_{3}$ - фермионное суперполе. Зададим новое бозонное суперполе $N$ и фермионное суперполе $h$, зависящие от $\Psi_{1}, \Psi_{2}$ и $\Psi_{3}$ :

$$
N=\frac{\Psi_{1}}{\Psi_{2}}, \quad h=\frac{\Psi_{3}}{\Psi_{2}} .
$$

Теперь можно записать пару Лакса (12) как пару уравнений Риккатти для суперполей

$$
\begin{array}{rlrl}
D_{x} N & =\frac{1}{\sqrt{\lambda}} h\left(-D_{x} Q+N D_{x} R\right), & D_{t} N=\left(1+N^{2}\right) R-2 \sqrt{\lambda} h, \\
D_{x} h=\frac{1}{2 \sqrt{\lambda}}\left(D_{x} Q N+D_{x} R\right), & D_{t} h=-h R N-\sqrt{\lambda} N .
\end{array}
$$

Эти соотношения далее используются как генераторы бесконечной последовательности уравнений непрерывности для суперполей. Введем суперполе

$$
W=\ln \Psi_{2} .
$$

Подействовав оператором $D_{x}$ на обе части этого равенства, с учетом пары Лакса (12) получим уравнение $D_{x} W=-\frac{1}{\sqrt{\lambda}} D_{x} R h$. Затем подействуем оператором $D_{t}$ и получим уравнение $D_{t} W=-R N$.

Условие совместности $D_{x} D_{t} W=-D_{t} D_{x} W$ теперь принимает вид закона сохранения для суперполей (уравнения непрерывности)

$$
D_{x} J(x, t, \theta, \zeta ; \lambda)=D_{t} K(x, t, \theta, \zeta ; \lambda),
$$

где суперполевые токи задаются как

$$
J(x, t, \theta, \zeta ; \lambda)=R N, \quad K(x, t, \theta, \zeta ; \lambda)=-\frac{1}{\sqrt{\lambda}} D_{x} R h .
$$

Из второго уравнения в (15) мы можем записать

$$
2 \sqrt{\lambda} h=-D_{t} N+\left(1+N^{2}\right) R, \quad \sqrt{\lambda} h=\frac{R}{2}+\frac{1}{2 \sqrt{\lambda}} \partial_{t} h+\frac{1}{2 \lambda} D_{t} R D_{t} h \cdot h .
$$

Разлагая суперполе $h$ в ряд по $\lambda$, мы приходим к бесконечному набору уравнений непрерывности. Пусть

$$
h=\frac{1}{\sqrt{\lambda}} \sum_{n=0}^{\infty} \frac{h^{(n+1 / 2)}}{\lambda^{n}} .
$$

Подставим это разложение в (19), получим рекуррентные уравнения $h^{(1 / 2)}=\frac{R}{2}, \quad h^{(n+1 / 2)}=\frac{1}{2} \partial_{t} h^{(n-1 / 2)}+\frac{1}{2} D_{t} R \sum_{l=1}^{n-1}\left(D_{t} h^{(l-1 / 2)}\right) h^{(n-l-1 / 2)}, \quad n=1,2, \ldots$ 
Поскольку $N=-D_{t} h / \sqrt{\lambda}$, теперь можно записать (18) как $D_{x}\left(R D_{t} h\right)=D_{t}\left(D_{x} R h\right)$; используя в получившемся уравнении разложение $(20)$, получаем следующие рекуррентные законы сохранения для суперполей:

$$
D_{x} J^{(n+1 / 2)}=D_{t} K^{(n+1 / 2)},
$$

где суперполевые токи $J^{(n+1 / 2)}$ и $K^{(n+1 / 2)}$ задаются как

$$
J^{(n+1 / 2)}=R D_{t} h^{(n-1 / 2)}, \quad K^{(n+1 / 2)}=D_{x} R h^{(n-1 / 2)} .
$$

Несколько первых токов имеют вид

$$
\begin{aligned}
& D_{x}\left(R D_{t} R\right)=D_{t}\left(D_{x} R \cdot R\right), \quad D_{x}\left(R D_{t} \partial_{t} R\right)=D_{t}\left(D_{x} R \partial_{t} R\right), \\
& D_{x}\left(R D_{t}\left[\partial_{t}^{2} R+\frac{1}{2}\left(D_{t} R\right)^{2} R\right]\right)=D_{t}\left(D_{x} R\left[\partial_{t}^{2} R+\frac{1}{2}\left(D_{t} R\right)^{2} R\right]\right) .
\end{aligned}
$$

\section{4. ПРЕОБРАЗОВАНИЕ ДАРБУ SUSY-CD-СИСТЕМЫ}

Преобразование Дарбу применяется для расчета мультисолитонных решений интегрируемых систем. Если заданы уравнения (1) для суперполей и их пара Лакса (6), можно получить такие мультисолитонные решения, как суперполя $\psi, \phi$ и $\chi$

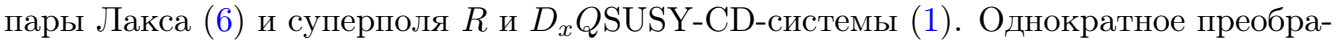
зование Дарбу для суперполей пары Лакса (6) имеет вид

$$
\begin{gathered}
\psi[1]=\lambda \phi-\lambda_{1} \frac{\phi_{1}}{\psi_{1}} \psi-\sqrt{\lambda \lambda_{1}} \frac{\chi_{1}}{\psi_{1}} \chi, \quad \phi[1]=\lambda \psi-\lambda_{1} \frac{\psi_{1}}{\phi_{1}} \phi-\sqrt{\lambda \lambda_{1}} \frac{\chi_{1}}{\phi_{1}} \chi, \\
\chi[1]=-\left(\lambda+\lambda_{1}\right) \chi-\sqrt{\lambda \lambda_{1}} \frac{\chi_{1}}{\psi_{1}} \psi-\sqrt{\lambda \lambda_{1}} \frac{\chi_{1}}{\phi_{1}} \phi
\end{gathered}
$$

где $\psi, \phi$ и $\chi$ - решения пары Лакса при произвольном значении спектрального параметра $\lambda$, а $\psi_{1}, \phi_{1}$ и $\chi_{1}$ - частные решения пары Лакса при значении спектрального параметра $\lambda_{1}$. Новые суперполя $\psi[1], \phi[1]$ и $\chi[1]$ удовлетворяют уравнениям той же пары Лакса (6) при $\lambda=\lambda_{1}$. Преобразование Дарбу суперполей $R$ и $D_{x} Q$ в терминах частных решений $\phi_{1}$ и $\psi_{1}$ задается как

$$
\begin{aligned}
R[1] & =-R-i D_{t} \ln \frac{\phi_{1}}{\psi_{1}} \\
D_{x} Q[1] & =-\frac{1}{2} D_{x} Q\left(\frac{\psi_{1}}{\phi_{1}}+\frac{\phi_{1}}{\psi_{1}}\right)+\frac{i}{2} D_{x} R\left(\frac{\phi_{1}}{\psi_{1}}-\frac{\psi_{1}}{\phi_{1}}\right),
\end{aligned}
$$

где $R[1]$ и $D_{x} Q[1]$ - новые решения уравнения (1), выраженные через частные решения пары Лакса.

Аналогично мы получаем следующие преобразования суперполя $R$ и производной $D_{x} Q$ :

$$
\begin{aligned}
R[1] & =R+i \sqrt{\lambda_{1}}\left(\frac{\chi_{1}}{\psi_{1}}+\frac{\chi_{1}}{\phi_{1}}\right) \\
D_{x} R[1] & =\frac{i}{2} D_{x} Q\left(\frac{\psi_{1}}{\phi_{1}}-\frac{\phi_{1}}{\psi_{1}}\right)-\frac{1}{2} D_{x} R\left(\frac{\phi_{1}}{\psi_{1}}+\frac{\psi_{1}}{\phi_{1}}\right) .
\end{aligned}
$$


Двукратные преобразования Дарбу для решений $R$ и $D_{x} Q$ выводятся путем повторного применения преобразования Дарбу, что дает следующий результат:

$$
\begin{aligned}
R[2]= & -R-i D_{t} \ln \frac{\Delta_{12}^{2}[2]+i \sqrt{\lambda_{1} \lambda_{2}} X_{12}}{\Delta_{12}^{1}[2]+i \sqrt{\lambda_{1} \lambda_{2}} X_{12}} \\
D_{x} Q[2]= & -\frac{1}{2} D_{x} Q\left(\frac{\Delta_{12}^{1}[2]+i \sqrt{\lambda_{1} \lambda_{2}} X_{12}}{\Delta_{12}^{2}[2]+i \sqrt{\lambda_{1} \lambda_{2}} X_{12}}+\frac{\Delta_{12}^{2}[2]+i \sqrt{\lambda_{1} \lambda_{2}} X_{12}}{\Delta_{12}^{1}[2]+i \sqrt{\lambda_{1} \lambda_{2}} X_{12}}\right)+ \\
& +\frac{i}{2} D_{x} R\left(\frac{\Delta_{12}^{2}[2]+i \sqrt{\lambda_{1} \lambda_{2}} X_{12}}{\Delta_{12}^{1}[2]+i \sqrt{\lambda_{1} \lambda_{2}} X_{12}}-\frac{\Delta_{12}^{1}[2]+i \sqrt{\lambda_{1} \lambda_{2}} X_{12}}{\Delta_{12}^{2}[2]+i \sqrt{\lambda_{1} \lambda_{2}} X_{12}}\right)
\end{aligned}
$$

Поле производной $D_{x} R$ преобразуется как

$$
\begin{aligned}
D_{x} R[2]=\frac{i}{2} & D_{x} Q\left(\frac{\Delta_{12}^{1}[2]+i \sqrt{\lambda_{1} \lambda_{2}} X_{12}}{\Delta_{12}^{2}[2]+i \sqrt{\lambda_{1} \lambda_{2}} X_{12}}-\frac{\Delta_{12}^{2}[2]+i \sqrt{\lambda_{1} \lambda_{2}} X_{12}}{\Delta_{12}^{1}[2]+i \sqrt{\lambda_{1} \lambda_{2}} X_{12}}\right)- \\
& -\frac{1}{2} D_{x} R\left(\frac{\Delta_{12}^{2}[2]+i \sqrt{\lambda_{1} \lambda_{2}} X_{12}}{\Delta_{12}^{1}[2]+i \sqrt{\lambda_{1} \lambda_{2}} X_{12}}+\frac{\Delta_{12}^{1}[2]+i \sqrt{\lambda_{1} \lambda_{2}} X_{12}}{\Delta_{12}^{2}[2]+i \sqrt{\lambda_{1} \lambda_{2}} X_{12}}\right) .
\end{aligned}
$$

В этих формулах

$$
\Delta_{12}^{1}[2]=\left|\begin{array}{cc}
\lambda_{1} \phi_{1} & \lambda_{2} \phi_{2} \\
\psi_{1} & \psi_{2}
\end{array}\right|, \quad \Delta_{12}^{2}[2]=\left|\begin{array}{cc}
\lambda_{1} \psi_{1} & \lambda_{2} \psi_{2} \\
\phi_{1} & \phi_{2}
\end{array}\right|, \quad X_{12}=\chi_{1} \chi_{2} .
$$

Чтобы объяснить детерминантную структуру мультисолитонных решений, рассмотрим пример троекратного преобразования Дарбу. Решения $R$ и $D_{x} Q$, получающиеся после применения этого преобразования, имеют вид

$$
\begin{gathered}
R[3]=-R-i D_{t} \ln \frac{\boldsymbol{\Delta}^{1}}{\boldsymbol{\Delta}^{2}} \\
D_{x} Q[3]=-\frac{1}{2} D_{x} Q\left(\frac{\boldsymbol{\Delta}^{1}}{\boldsymbol{\Delta}^{2}}+\frac{\boldsymbol{\Delta}^{2}}{\boldsymbol{\Delta}^{1}}\right)+\frac{i}{2} D_{x} R\left(\frac{\boldsymbol{\Delta}^{1}}{\boldsymbol{\Delta}^{2}}-\frac{\boldsymbol{\Delta}^{2}}{\boldsymbol{\Delta}^{1}}\right)
\end{gathered}
$$

где

$$
\begin{aligned}
\boldsymbol{\Delta}^{k}=\Delta_{123}^{k}[3] & +i \sqrt{\lambda_{2} \lambda_{3}}\left(\lambda_{1}+\lambda_{2}\right)\left(\lambda_{1}+\lambda_{3}\right) \Delta_{1}^{k}[1] X_{23}+ \\
& +i \sqrt{\lambda_{1} \lambda_{3}}\left(\lambda_{1}+\lambda_{2}\right)\left(\lambda_{2}+\lambda_{3}\right) \Delta_{2}^{k}[1] X_{13}+ \\
& +i \sqrt{\lambda_{1} \lambda_{2}}\left(\lambda_{1}+\lambda_{3}\right)\left(\lambda_{2}+\lambda_{3}\right) \Delta_{3}^{k}[1] X_{12}, \quad k=1,2,
\end{aligned}
$$

и

$$
\begin{gathered}
\Delta_{123}^{1}[3]=\left|\begin{array}{ccc}
\lambda_{1}^{2} \psi_{1} & \lambda_{2}^{2} \psi_{2} & \lambda_{3}^{2} \psi_{3} \\
\lambda_{1} \phi_{1} & \lambda_{2} \phi_{2} & \lambda_{3} \phi_{3} \\
\psi_{1} & \psi_{2} & \psi_{3}
\end{array}\right|, \quad \Delta_{123}^{2}[3]=\left|\begin{array}{ccc}
\lambda_{1}^{2} \phi_{1} & \lambda_{2}^{2} \phi_{2} & \lambda_{3}^{2} \phi_{3} \\
\lambda_{1} \psi_{1} & \lambda_{2} \psi_{2} & \lambda_{3} \psi_{3} \\
\phi_{1} & \phi_{2} & \phi_{3}
\end{array}\right|, \\
\Delta_{j}^{1}[1]=\psi_{j}, \quad \Delta_{j}^{2}[1]=\phi_{j}, \quad X_{\ell j}=\chi_{\ell} \chi_{j}, \quad \ell, j=1,2,3 .
\end{gathered}
$$


Если $N$ раз применить преобразование Дарбу, получается $N$-солитонное решение. При нечетных $N$ оно имеет вид

$$
R[N]=-R-i D_{t} \ln \frac{\sum_{m=0}^{(n+1) / 2} i^{m} P\left(\lambda_{k_{j}} ; \lambda_{k_{v}}\right) \Delta_{k_{j}}^{1} X_{k_{v}}}{\sum_{m=0}^{(n+1) / 2} i^{m} P\left(\lambda_{k_{j}} ; \lambda_{k_{v}}\right) \Delta_{k_{j}}^{2} X_{k_{v}}},
$$

а при четных $N$ записывается как

$$
R[N]=-R-i D_{t} \ln \frac{\sum_{m=0}^{n / 2} i^{m} P\left(\lambda_{k_{j}} ; \lambda_{k_{v}}\right) \Delta_{k_{j}}^{1} X_{k_{v}}+\sum_{m=1}^{n / 2-1} P\left(\lambda_{k_{j}} ; \lambda_{k_{v}}\right) \frac{(-1)^{m}}{m !} X_{k_{j}} X_{k_{v}}}{\sum_{m=0}^{n / 2} i^{m} P\left(\lambda_{k_{j}} ; \lambda_{k_{v}}\right) \Delta_{k_{j}}^{2} X_{k_{v}}+\sum_{m=1}^{n / 2-1} P\left(\lambda_{k_{j}} ; \lambda_{k_{v}}\right) \frac{(-1)^{m}}{m !} X_{k_{j}} X_{k_{v}}},
$$

где $k_{j}$ обозначает набор из $n-2 m$ упорядоченных индексов, $k_{v}$ обозначает набор остальных $2 m$ упорядоченных индексов из $\{1, \ldots, n\}$ и введены обозначения

$$
\Delta_{a b c \ldots}^{1}=\left|\begin{array}{cccc}
\vdots & \vdots & \vdots & . \cdot \\
\lambda_{a}^{2} \psi_{a} & \lambda_{b}^{2} \psi_{b} & \lambda_{c}^{2} \psi_{c} & \ldots \\
\lambda_{a} \phi_{a} & \lambda_{b} \phi_{b} & \lambda_{c} \phi_{c} & \ldots \\
\psi_{a} & \psi_{b} & \psi_{c} & \ldots
\end{array}\right|, \quad \Delta_{a b c \ldots}^{2}=\left|\begin{array}{cccc}
\vdots & \vdots & \vdots \\
\lambda_{a}^{2} \phi_{a} & \lambda_{b}^{2} \phi_{b} & \lambda_{c}^{2} \phi_{c} & \ldots \\
\lambda_{a} \psi_{a} & \lambda_{b} \psi_{b} & \lambda_{c} \psi_{c} & \ldots \\
\phi_{a} & \phi_{b} & \phi_{c} & \ldots
\end{array}\right|
$$

И

$$
\begin{gathered}
X_{a b c \ldots}=\sqrt{\lambda_{a} \lambda_{b} \lambda_{c} \ldots} \chi_{a} \chi_{b} \chi_{c} \ldots \\
P\left(\lambda_{a}, \lambda_{b}, \ldots ; \lambda_{c}, \lambda_{d}, \ldots\right)=(-1)^{N+\alpha}\left(\lambda_{a}+\lambda_{c}\right)\left(\lambda_{a}+\lambda_{d}\right) \ldots\left(\lambda_{b}+\lambda_{c}\right)\left(\lambda_{b}+\lambda_{d}\right) \ldots .
\end{gathered}
$$

Здесь $P\left(\lambda_{k_{j}} ; \varnothing\right)=P\left(\varnothing ; \lambda_{k_{v}}\right)=1$ (т. е. $P=1$, если слева или справа от точки с запятой в аргументе функции нет $\left.\lambda_{k}\right)$ и $\alpha$ - бинарная функция, которая равна нулю, если $a b \ldots c d \ldots$ - четная циклическая перестановка индексов $1, \ldots, n$, и равна единице в случае нечетной перестановки.

На следующем шаге мы получаем явные решения SUSY-CD-системы. Начнем с затравочного решения $D_{x} Q=1, R=0$. Уравнения (22) в этом случае принимают вид

$$
R[1]=i D_{t} \ln \frac{\psi_{1}}{\phi_{1}}, \quad D_{x} Q[1]=-\frac{1}{2}\left(\frac{\psi_{1}}{\phi_{1}}+\frac{\phi_{1}}{\psi_{1}}\right) .
$$

Выберем $\psi_{1}$ и $\phi_{1}$ как

$$
\psi_{1}=e^{\zeta \theta / 2}-e^{(k x+w t)-(\xi+\zeta)(\theta+\lambda) / 2}, \quad \phi_{1}=e^{\zeta \theta / 2}+e^{(k x+w t)-(\xi+\zeta)(\theta+\lambda) / 2} .
$$

Оказывается, что они являются решениями уравнений (23), если выполнены бозонное дисперсионное соотношение $w=-1 / k$ и фермионное дисперсионное соотношение $\lambda=k \xi$. Подставляя функции (24) в уравнения (23), мы получаем решения SUSY-CD-системы

$$
R[1]=-i \vartheta \operatorname{csch} 2 \beta, \quad D_{x} Q[1]=-\operatorname{cth} 2 \beta,
$$

где

$$
2 \beta=\frac{\zeta \theta}{2}+\frac{(\xi+\zeta)(\theta+\lambda)}{2}-(k x+w t), \quad \vartheta=\zeta w-\theta-\frac{\lambda}{2} .
$$

Фермионный вклад в $\beta$ появился благодаря суперсимметрии. 


\section{5. ЗАКЛЮЧИТЕЛЬНЫЕ ЗАМЕЧАНИЯ}

В представленной статье мы рассмотрели различные свойства интегрируемости, связанные с $N=1$ SUSY-CD-системой. Для этой системы мы ввели суперполя, выраженные в терминах суперматриц из супералгебры $\mathfrak{s}(2 \mid 1, \mathcal{G})$, и показали, что условие нулевой кривизны эквивалентно SUSY-CD-системе. Из пары Лакса для суперполей мы получили связанные пары суперполевых уравнений Риккати, которые затем использовали для построения бесконечного набора сохраняющихся суперполевых токов. Также было исследовано преобразование Дарбу для SUSY-CD-системы, которое мы применили для получения $N$-суперсолитонного решения системы в терминах вронскианов.

\section{Список литературы}

[1] Ю. Весс, Дж. Беггер, Суперсимметрия и супергравитачия, Мир, М., 1986.

[2] J. Wess, B. Zumino, "Supergauge transformations in four dimensions", Nucl. Phys. B, 70:1 (1974), 39-50.

[3] A. Salam, J. Strathdee, "Super-gauge transformations", Nucl. Phys. B, 76:3 (1974), $477-482$.

[4] P. Mathieu, "Supersymmetric extension of the Korteweg-de Vries equation", J. Math. Phys., 29:11 (1988), 2499-2506.

[5] Yu. I. Manin, A. O. Radul, "A supersymmetric extension of the Kadomtsev-Petviashvili hierarchy", Commun. Math. Phys., 98:1 (1985), 65-77.

[6] G. H. M. Roelofs, P. H. M. Kersten, "Supersymmetric extensions of the nonlinear Schrödinger equation: symmetries and coverings", J. Math. Phys., 33:6 (1992), 2185-2206.

[7] P. G. Drazin, R. S. Johnson, Solitons: An Introduction, Cambridge Univ. Press, Cambridge, 1989.

[8] Q.P. Liu, "Darboux transformations for supersymmetric Korteweg-de Vries equations", Lett. Math. Phys., 35:2 (1995), 115-122.

[9] Q. P. Liu, M. Mañas, "Darboux transformation for the Manin-Radul supersymmetric KdV equation", Phys. Lett. B, 394:3-4 (1997), 337-342.

[10] Q.P. Liu, M. Mañas, "Crum transformation and Wronskian type solutions for supersymmetric KdV equation", Phys. Lett. B, 396:1-4 (1997), 133-140.

[11] Q.P. Liu, M. Mañas, "Pfaffian solutions for the Manin-Radul-Mathieu SUSY KdV and SUSY sine-Gordon equations", Phys. Lett. B, 436:3-4 (1998), 306-310.

[12] C. Gu, H. Hu, Z. Zhou, Darboux Transformations in Integrable Systems. Theory and their Applications to Geometry, Mathematical Physics Studies, 26, Springer, Dordrecht, 2005.

[13] M. Siddiq, M. Hassan, U. Saleem, "On Darboux transformation of the supersymmetric sine-Gordon equation", J. Phys. A: Math. Gen., 39:23 (2006), 7313-7318.

[14] M. Hassan, "Darboux transformation of the generalized coupled dispersionless integrable system", J. Phys. A: Math. Theor., 42:6 (2009), 065203, 11 pp.

[15] U. Saleem, M. Hassan, "Darboux transformation and exact multisolitons of $\mathbb{C} P^{N}$ nonlinear sigma model", J. Math. Anal. Appl., 447:2 (2017), 1080-1101.

[16] N. Mushahid, M. ul Hassan, "A noncommutative coupled dispersionless system. Darboux transformation and explicit solutions", Modern. Phys. Lett. A, 29:39 (2014), 1450206, 20 pp.

[17] A. Mirza, M. ul Hassan, "Bilinearization and soliton solutions of the $N=1$ supersymmetric coupled dispersionless integrable system", J. Nonlinear Math. Phys., 24:1 (2017), 107-115.

[18] K. Konno, H. Oono, "New coupled integrable dispersionless equations", J. Phys. Soc. Japan, 63:2 (1994), 377-378.

[19] R. Hirota, S. Tsujimoto, "Note on 'New coupled integrable dispersionless equations'", J. Phys. Soc. Japan, 63:9 (1994), 3533. 
[20] H. Kakuhata, K. Konno, "A generalization of coupled integrable, dispersionless system", J. Phys. Soc. Japan, 65:2 (1996), 340-341.

[21] H. Kakuhata, K. Konno, "Lagrangian, Hamiltonian and conserved quantities for coupled integrable dispersionless equations", J. Phys. Soc. Japan, 65:1 (1995), 1-2.

[22] H. Kakuhata, K. Konno, "Canonical formulation of generalized coupled dispersionless system", J. Phys. A: Math. Gen., 30:12 (1997), L401-L408.

[23] T. Alagesan, K. Porsezian, "Painlevé analysis and the integrability properties of coupled integrable dispersionless equations", Chaos, Solitons Fractals, 7:8 (1996), 1209-1212.

[24] T. Alagesan, K. Porsezian, "Singularity structure analysis and Hirota's bilinearisation of the coupled integrable dispersionless equations", Chaos, Solitons Fractals, 8:10 (1997), $1645-1650$.

[25] T. Alagesan, Y. Chung, K. Nakkeeran, "Bäcklund transformation and soliton solutions for the coupled dispersionless equations", Chaos, Solitons Fractals, 21:1 (2004), 63-67.

[26] A. Chen, X. Li, "Soliton solutions of the coupled dispersionless equation", Phys. Lett. A, 370:3-4 (2007), 281-286.

[27] N. Mushahid, M. ul Hassan, "On the dressing method for the generalized coupled dispersionless integrable system", Modern Phys. Lett. A, 28:20 (2013), 1350088, 20 pp.

[28] M. Siddiq, M. Hassan, "On the linearization of the super sine-Gordon equation", Europhys. Lett., 70:2 (2005), 149-154.

[29] S. Bertrand, A. M. Grundland, A. J. Hariton, "On the integrability of supersymmetric versions of the structural equations for conformally parametrized surfaces", SIGMA, 11 (2015), 046, 16 pp.

[30] M. Wadati, H. Sanuki, K. Konno, "Relationship among inverse method, Bäcklund transformation and an infinite number of conservation laws", Progr. Theor. Phys., 53:2 (1975), 419-436.

[31] S. Ferrera, L. Girardello, S. Sciuto, "An infinite set of conservation laws of the supersymmetric sine-Gordon theory", Phys. Lett. B, 76:3 (1978), 303-306.

[32] L. Girardello, S. Sciuto, "Inverse scattering-like problem for supersymmetric models", Phys. Lett. B, 77:3 (1978), 267-269.

Поступила в редакцию 23.10.2017, после доработки 12.12.2017 